\title{
La MiniQuest a la vanguardia en Tecnologías para el Aprendizaje
}

\section{MiniQuest at the forefront of Learning Technologies}

\author{
DOI: $10.46932 / \mathrm{sfjdv} 2 \mathrm{n} 4-065$
}

Received in: March 1st, 2021

Accepted in: May 30th, 2021

\author{
Aurora Farfán Márquez \\ Maestra en Ciencias. Área de Concentración de Salud Reproductiva. INSP \\ Lic. en Etnología. Escuela Nacional de Antropología e Historia. \\ Universidad Nacional Autónoma de México. Facultad de Medicina.UNAM \\ Programa de Alta Exigencia Académica. \\ Circuito Escolar 411A, Copilco Universidad, Coyoacán, 04360 Ciudad de México, CDMX \\ E- mail: aurora_fa_ma@hotmail.com \\ Rosa María Zúñiga Sánchez \\ Medica Cirujana. Egresada de la UNAM \\ Maestría en educación médica, Westhill \\ Profesora de la Escuela Nacional Preparatoria UNAM \\ Circuito Escolar 411A, Copilco Universidad, Coyoacán, 04360 Ciudad de México, CDMX \\ E-mail roszauniga@gmail.com

\section{Emilia Cantoral Farfán} \\ Médico especialista en Nefrología, egresada de Centro Médico Nacional Siglo XXI, Instituto Mexicano \\ del Seguro Social. \\ Hospital General de Zona 32, IMSS y Facultad de Medicina UNAM \\ Circuito Escolar 411A, Copilco Universidad, Coyoacán, 04360 Ciudad de México, CDMX \\ E- mail: emilia.cantoral@icloud.com
}

\section{RESUMEN}

Introducción

Las tecnologías de la información tienen alrededor de tres décadas ayudándonos a realizar trabajos más rápidos, creativos y lo más sorprendente colaborativos ya que podemos estar en diferentes lugares al mismo tiempo y mantener la comunicación. En esta época actual el internet, resulta útil y acorta distancias. Ello es un estímulo para integrar la enseñanza-aprendizaje y realizar mejoras en el aula dejando de lado la enseñanza tradicional. Mediante el internet, podemos diseñar y planear diversos cursos y trabajar con ellos sobre proyectos e investigaciones que ellos mismos propongan, diseñen, estructuren y en ocasiones puedan publicar, complementando las actividades anteriormente propias del aula.

Objetivo

Construcción del aprendizaje situado mediante la MiniQuest como una estrategia metodológica, como lo es el aprendizaje basado en problemas (ABP).

Población objeto:

Grupo de 40 alumnos de primer año de la carrera de médico cirujano, del ciclo escolar 2017-2018 de la Facultad de medicina de la UNAM.

Aporte principal del trabajo:

Los alumnos con este tipo de estrategias metodológicas miniquest, podrán desarrollar algunas de las competencias del Plan de estudios 2010 como son pensamiento crítico, desarrollar razonamiento médico, 
toma de decisiones y fomentar las habilidades para la investigación. Además proporcionar los elementos necesarios para que desarrollen el profesionalismo médico, en su vida futura.

Palabras clave: TIC, MiniQuest, Aprendizaje, Estrategias de aprendizaje

\begin{abstract}
Introduction

Information technologies have been helping us for about three decades to do faster, creative and most surprisingly collaborative work since we can be in different places at the same time and maintain communication. In this current era the internet is useful and shortens distances. This is a stimulus to integrate teaching-learning and make improvements in the classroom leaving aside the traditional teaching. Through the Internet, we can design and plan various courses and work with them on projects and research that they themselves propose, design, structure and sometimes can publish, complementing the activities previously typical of the classroom.

Objective

Construction of situated learning through MiniQuest as a methodological strategy, as is problem-based learning (PBL).

Target population:

Group of 40 first year students of the career of medical surgeon, of the 2017-2018 school cycle of the Faculty of Medicine of the UNAM.

Main contribution of the work:

Students with this type of miniquest methodological strategies, will be able to develop some of the competencies of the 2010 Curriculum such as critical thinking, develop medical reasoning, decision making and promote research skills. In addition to providing the necessary elements to develop medical professionalism in their future life.
\end{abstract}

Key words: ICT, MiniQuest, Learning, Learning strategies.

\title{
1 INTRODUCCIÓN
}

Dentro de los avances científicos del siglo XX, tenemos a las Tecnologías de la Información y Comunicación, definidas como un conjunto de tecnologías que permiten el acceso, la producción, el tratamiento y la comunicación. Están representada en diferentes códigos tales como acceso, textos e imágenes entre los principales. No obstante, el internet surge como la herramienta innovadora de cómo se relaciona el hombre en la actualidad.

Ahora para tener acceso a dicha información lo hacemos mediante los recursos informáticos y telemáticos. No obstante, el triunfo de la web se debe a un protocolo de comunicación y un lenguaje tipo a lo que le siguió un sin número de usuarios en este nuevo entorno cada vez con mayor número de funcionalidades.

El empleo de las tecnologías de la información desde su origen ha sido una alternativa de apoyo para los docentes ayudando a realizar las tareas y trabajos académicos más rápidos y mejorando sus métodos de enseñanza, con creatividad y de manera colaborativos esto es, al mismo tiempo podemos estar en diferentes lugares y mantenemos la comunicación. En esta época actual con internet, resulta 
útil acortar distancias. Esto se convierte en un estimulo para el profesor que lo puede integrar en la enseñanza-aprendizaje y realizar mejoras en el aula dejando de lado la enseñanza tradicional. Con el internet, podemos diseñar y planear diversos cursos y trabajar con ellos sobre proyectos e investigaciones que ellos mismos eligen, diseñan, estructuran y en ocasiones publican, complementando de esta manera su actividad del aula. De tal manera que la comunicación, como el elemento inmerso en la sociedad es usado en la práctica de la enseñanza-aprendizaje (Mendoza, 2021).

\section{DESARROLLO}

En la actualidad las TIC, son un excelente soporte para la creación de entornos de aprendizaje abiertos esto es favorecen la labor del docente en el aula, transformando el proceso educativo de ser pasivo centrado en el profesor a ser activo con la participación de los alumnos en su aprendizaje. Para ello, se requiere de docentes que estén capacitados y que saben navegar en internet para adquirir conocimientos, además del empleo de las herramientas de información para preparar alumnos aplicando nuevas técnicas y métodos de educación promovidos por la tecnología.

No obstante, el gran reto es que estas nuevas tecnologías, puedan ser empleadas para mejorar la educación de la población y que se difundan al resto de la sociedad. Ello involucra el desarrollo de la comunicación y sus formas de comunicarnos que se han desarrollado de manera impresionante y puede contribuir a disminuir la desigualdad social en lo que a educación se refiere ya que se puede hacer llegar esta a los lugares donde la infraestructura es escasa o nula y donde los caminos son muy accidentados o no existen. Esta comunicación educativa se ha visto obligada a tener una actualización continua y estar a la vanguardia de las nuevas tecnologías de información y comunicación TIC.

Sin embargo, el empleo de las TIC se ha difundido en las últimas décadas, es indispensable conocer como ha influido en la educación y cómo hoy día influye en los diversos niveles educativos y en cual, ha tenido mayor aplicación, desarrollo y uso por sus actores. Por ejemplo, el INEGI en 2011, señalo que son los adolescentes de entre 12 y 24 años en donde las TIC son más empleadas; es que en esta población se plantea su uso en la vida cotidiana.

De ahí que se proponen el aprendizaje situado, como una estrategia educativa son nada menos que escenarios o casos clínicos en forma de narrativa, con los que los estudiantes (actores) se van a enfrentar durante su desarrollo profesional. Y el profesor no requiere experiencia para aplicarla (Adell, 2009). Pero en si son pequeños contextos de la realidad en donde desarrollan varias competencias educativas tales como pensamiento crítico, ya que entre ellos sea en aula o en un foro se discute acerca del caso clínico involucrándose en él se destacan la expresión y educación de emociones y valores; el razonamiento medico, porque ellos deben incluir las asignaturas se primer año para que puedan discernir mezclando los 
aprendizajes cognitivos y afectivos previos y, posteriormente tomar de decisiones, sea en el diagnóstico, el tratamiento y la terapéutica a seguir sin dejar de lado la ética profesional. Por ello, este tipo de aprendizaje situado se centra en el alumno quien debe ser participativo en todo momento y responsable de su propio aprendizaje.

No obstante, el empleo de las TIC también tiene su lado negativo por ejemplo, en la impartición de justicia y promueve la acumulación del poder en unos cuantos, tal es el caso de las grandes empresas de comunicación (Belloch, 2014).

La importancia de las TIC y su uso diario, así como, el empleo de estas en la enseñanza con respecto al rendimiento académico. Bajo esta perspectiva podemos deducir que las TIC son la fuente principal para la construcción del conocimiento crítico y reflexivo. El empleo de los jóvenes obliga a cambiar los modelos de aprendizaje tradicionales por una perspectiva integradora. En donde el desempeño de los alumnos está dirigido a la búsqueda de información en fuentes fidedignas fortaleciendo su pensamiento crítico.

El que los alumnos cuenten en sus hogares con internet, nos ayuda a planear diversos cursos y trabajar con ellos sobre proyectos e investigaciones que ellos mismos eligen, diseñan, estructuran y en ocasiones publican, complementando así su actividad que anteriormente correspondía solo al aula. Por ejemplo, la estrategia metodológica del aprendizaje basado en problemas (ABP) en estudiantes de la Licenciatura de médico cirujano en la UNAM.

Se diseño un ejercicio y empleo la Miniquests con los estudiantes de la carrera de medicina. Basado en la metodología de Aprendizaje Basado en Problemas (ABP) con el propósito de que los alumnos desarrollen el aprendizaje colaborativo y el razonamiento clínico.

Para el desarrollo del trabajo se tomo como base a un grupo de primer año de la licenciatura de médico cirujano y se les explico en qué consistía la técnica de miniquests y lo que aportaría en su aprendizaje a ellos como alumnos y a nosotras como sus profesoras. El total de los alumnos (40) aceptaron participar en el ejercicio. Se solicitó que tuvieran internet, ya sea en casa o en la escuela para que pudieran realizar las actividades. Se organizaron en grupos, se les asignaron tareas y tuvieron que elaborar un producto que va desde una presentación, un documento, hasta una escenificación teatral o un guion radiofónico.

En este trabajo exponemos una experiencia con un grupo de alumnos en donde se trabajó la miniquest durante los últimos seis meses del año escolar, esto fue con la finalidad de que en el primer semestre obtuvieran los elementos teóricos-metodológicos para que en la segunda parte del año desarrollen el pensamiento crítico y razonamiento médico. Para lo cual, se diseñó un ejercicio de miniquest, para la asignatura de anatomía sobre el tema de Métodos anticonceptivos, se prefirió esta 
estrategia ya que es dinámica los alumnos se agrupan en equipos ya que el número del grupo es grande de esta forma se facilita la supervisión y el manejo del tema en los alumnos. También, el tener tareas diseñadas por sesiones con objetivos muy claros y que sean posibles de realizar. La MiniQuest consiste de tres elementos:

a) Escenario es donde se plantea el problema con un recordatorio de los temas iniciales;

b) Tarea en este apartado se incluye una guía estructurada con instrucciones precisas, una pregunta que los alumnos deben responder partiendo de una pregunta inicial.

c) El Producto, es la descripción detallada sobre la respuesta inicial, en esta se observa la investigación que el alumno realizó sobre el tema y debe ser con respecto al escenario.

Para observar y evaluar su desempeño se apoya en una rúbrica como el siguiente ejemplo de Rúbrica:

\begin{tabular}{|c|c|c|c|c|c|}
\hline \multicolumn{6}{|c|}{ Rúbrica para Evaluar MiniQuest } \\
\hline $\begin{array}{c}\text { Aspectos a } \\
\text { evaluar }\end{array}$ & 1 & 2 & 3 & 4 & 5 \\
\hline \multirow[t]{2}{*}{ Introducción } & $\begin{array}{l}\text { No introduce las } \\
\text { generalidades del } \\
\text { tema; ni plantea el } \\
\text { objetivo. No } \\
\text { describe el } \\
\text { contenido del } \\
\text { mismo, ni específica } \\
\text { la forma en que se } \\
\text { hizo. }\end{array}$ & & $\begin{array}{l}\text { Introduce el tema } \\
\text { central, encuentra el } \\
\text { Objetivo. Describe el } \\
\text { contenido y enlista los } \\
\text { pasos que siguió para } \\
\text { su desarrollo }\end{array}$ & & $\begin{array}{l}\text { Introduce generalidades del tema } \\
\text { central; hace explicito el objetivo } \\
\text { y describe el contenido del mismo. } \\
\text { Explica los pasos que siguió para } \\
\text { realizar el trabajo y menciona las } \\
\text { conclusiones. }\end{array}$ \\
\hline & 1 & 2 & $\mathbf{3}$ & 4 & 5 \\
\hline $\begin{array}{c}\text { Desarrollo del } \\
\text { tema }\end{array}$ & $\begin{array}{l}\text { No presenta } \\
\text { resultados con base } \\
\text { en el marco de } \\
\text { análisis ni en la } \\
\text { bibliografía } \\
\text { relevante sobre el } \\
\text { tema. }\end{array}$ & & 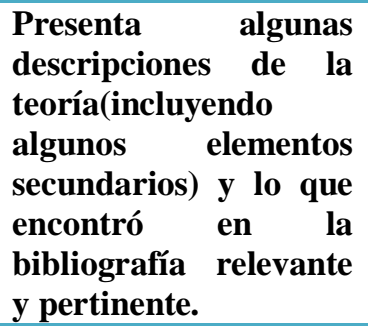 & & $\begin{array}{l}\text { Fundamenta los hallazgos y los } \\
\text { elementos de la teoría, lo cual le } \\
\text { sirve de evidencia para respaldar } \\
\text { la respuesta a la pregunta- } \\
\text { problema. }\end{array}$ \\
\hline $\begin{array}{l}\text { Resultado y } \\
\text { Argumentación }\end{array}$ & $\begin{array}{l}\text { Presenta opciones } \\
\text { centrales ron } \\
\text { sentido común. }\end{array}$ & & $\begin{array}{l}\text { Algunos de los } \\
\text { resultados } \\
\text { sustentados están } \\
\text { evidencias, otros no se } \\
\text { fundamentan. }\end{array}$ & & $\begin{array}{l}\text { Los resultados están sustentados } \\
\text { con evidencias científicas. }\end{array}$ \\
\hline Conclusión & $\begin{array}{l}\text { No plantea una } \\
\text { conclusión }\end{array}$ & & $\begin{array}{l}\text { Su conclusión esta } \\
\text { fundamentada en los } \\
\text { resultados }\end{array}$ & & $\begin{array}{l}\text { Su conclusión está fundamentada } \\
\text { en los resultados y hace una } \\
\text { reflexión sobre la literatura } \\
\text { científica consultada. }\end{array}$ \\
\hline
\end{tabular}




\section{I.Instrucción:}

\section{EJERCICIO DE UNA MINIQUEST PARA ALUMNOS}

$\checkmark \quad$ Elaboración de mapas conceptual y mental de los métodos anticonceptivos,

$\checkmark \quad$ En una tabla anotar la función, las indicaciones y las contraindicaciones de cada uno de los métodos anticonceptivos,

$\checkmark$ Conclusiones por subgrupo

$\checkmark$ Deben revisar la rúbrica para que su trabajo cumpla con lo requerido

\section{II.Trabajo en equipo}

- En plataforma Moodle:

- Búsqueda de información,

- Compartir experiencias

\section{III.Planeación}

(C) Objetivo: Planificación Familiar

(C) Contenido: Métodos Anticonceptivos

(e) Recuperación de ideas previas: anatomía y fisiología de los sistemas de la reproducción

Responder las siguientes preguntas

1. ¿Qué son los métodos anticonceptivos?

2. ¿Cómo se clasifican los métodos anticonceptivos?

3. ¿Cuáles son las funciones, indicaciones y contraindicaciones de cada uno de los métodos anticonceptivos?

Sesión 1. Plataforma virtual

(e) Se forman equipos de 5 alumnos

(C) En la plataforma virtual se coloca el mini caso:

(e) Rebeca N.

(e) "Femenino de 16 años, cursa $5^{\circ}$ años del bachillerato refiere que desea iniciar vida sexual con su novio, necesita saber que método anticonceptivo puede utiliza.

Rebeca pesa $70 \mathrm{Kg}$., con estatura de 1.55 mts."

Sesión 2: En el aula

Los alumnos deben anotar en el pizarrón:

$\checkmark \quad$ Las pistas del caso

$\checkmark$ Se proyectará los mapas conceptuales solicitados en la plataforma

$\checkmark \quad$ Discusión de los trabajos de cada equipo

$\checkmark \quad$ Elaboración de conclusiones del caso

\section{* Entregar un reporte de la actividad}

Sesión 3: en el aula

$\checkmark \quad$ Entregar un reporte (mapas conceptuales y mentales, la tablas)

$\checkmark \quad$ Elaboración de conclusiones por equipo y grupales. Evaluaciones a través de una rúbrica

* Entregar un reporte final de las dos sesiones 


\section{CONCLUSIONES}

Las TIC e internet, han introducido en los entornos educativos convencionales provocando una evolución hacia sistemas mixtos de enseñanza que mezclan lo presencial con lo virtual. El conocimiento se basa en los procesos de análisis, síntesis, evaluación e integración de la información.

Observar el impacto, que generan las TIC en la construcción del aprendizaje por ejemplo, en el área de la de la medicina es sorprendente ya que los alumnos crean su proceso de enseñanza aprendizaje desde una manera más lúdica y menos teórica lo que hace que el conocimiento que quede en su memoria de largo plazo ya que recordaran lo aprendido con pequeñas señales que se les presenten y no tiene que memorizar ya que ello es más fácil de olvidar porque queda en la memoria e corto plazo. Hay que reconocer que las generaciones de jóvenes actuales están al día en tecnologías y la enseñanza, por ello se debe dar este gran paso de lo tradicional al empleo de las TIC. Incluso para el docente, es otra forma de enseñar y se siente satisfecho por los resultados obtenidos. La anexión de las tecnologías en la educación, es un reclamo de la sociedad. Las ventajas y posibilidades que las TIC, aportan con su inserción a la formación favoreciendo el aprendizaje independiente facilitando el proceso enseñanza-aprendizaje (Toala, 2021).

Ya que ahora, diseña un aula virtual con apoyo de la tecnología la MiniQuest, por otra parte, realiza prácticas y experiencias de clase relacionadas con la utilización de esta. Así el proceso, enseñanzaaprendizaje en la medicina asegura el pensamiento crítico y el razonamiento médico, profesionales que se requiere hoy en día. A lo que debemos también tener en cuenta y considerar las implicaciones económicas políticas y culturales que su introducción implica.

Con este ejercicio los profesores percibieron la necesidad de actualizarse en cuanto a la forma de impartir los diversos temas la asignatura con el uso de las TIC. Para facilitar la evaluación, se diseñó la rúbrica con categorías e indicadores que nos permitieron medir resultados y analizar la información (Pérez, 2008). El estudio mezcla la parte del docente y sus estrategias pedagógicas con el uso de las TIC.

El mayor reto que enfrentamos fue el uso de plataformas virtuales (aula moodle) desarrollar procesos paralelos dentro de las actividades de enseñanza-aprendizaje cursos de actualización y formación para docentes además del diseño y elaboración de materiales educativos que puedan ser utilizados por la toda la comunidad. Encontrar una forma de integrar tecnologías al proceso de enseñanza-aprendizaje apoyar cursos presenciales. (Rivera, 2011) Integrar los aspectos tecnológicos como herramientas en el proceso educativo para la producción de materiales que coadyuven al proceso de enseñanza y que la brecha entre la enseñanza tradicional se transforme en la que es centrada en el alumno. 
Por lo anterior, la miniquest considerada como un modelo de estrategia que pretende beneficiar y minimizar el tiempo de los estudiantes, centrarse en el uso de la información más que en su búsqueda y refuerza los procesos intelectuales en los niveles de análisis, síntesis y evaluación. 


\section{REFERENCIAS}

1. Adell, Jordi. (2009). "WebQuest para aprender". Revista digital para profesionales de la enseñanza, 17, (p.1-47). https://docplayer.es/20284918-Webquest-para-aprender.html (Consulta 27/08/2018).

2. $\quad$ Area Moreira Manue. (2018). "WEBQUEST. Una estrategia de aprendizaje por descubrimiento basada en el uso de internet". Laboratorio de Educación y Nuevas Tecnologías, 1, (p.1-6). http://roble.pntic.mec.es/ atejero/recursos/presentawq.pdf

(Consulta 27/08/2018).

3. Belloch Ortí, Consuelo (2014). "Las Tecnologías de la Información y Comunicación (T.I.C.)". Unidad de Tecnología Educativa. Universidad de Valencia. http://www.uv.es/ bellochc/pdf/pwtic1.pdf. (Consulta 26/08/2018).

4. EDUTEKA. (2018). "Construyendo una MiniQuest. 8 junio 2018, de EDUTEKA - Tecnologías de Información y Comunicaciones para Enseñanza Básica y Media http://www.biopoint.com/miniquests/miniquests.html (Consulta 27/08/2018).

5. Mendoza A. Gricelia L. Santana Sardi Gustavo; Mawyin Cevallos Francisco A. (2021). Estrategias de la comunicación educativa en las tic, cómo alternativa de apoyo en el contexto universitario ecuatoriano. South Florida Journal of Development, Miami,Vol. 2 No. 2 apr/jun.2021

6. Pérez, R (2008) “Aportes de la Multimedia al Proceso de Enseñanza y Aprendizaje” Módulo de Salud Integral. en Revista ciencias de la educación, ISSN 1316-5917, No.31 https://dialnet.unirioja.es/servlet/articulo?codigo=5364413 (Consulta 27/08/2018).

7. Rivera M., Jorge (2011). "Impacto de las tecnologías de información y comunicación en los procesos de enseñanza-aprendizaje”. Investigación Educativa Vol.15 No.27 (pp.127-137)

http://sisbib.unmsm.edu.pe/BibVirtual/Publicaciones/Inv_Educativa/2011_n27/a08v15n27.pdf

(Consulta 30/08/2018).

8. Swig Sarah (2015). "Tics y Formación inicial y desarrollo profesional docente" Notas de política preal. Interamerican Dialogue. (p.1-8) recursos.portaleducoas.org/sites/default/files/349.pdf (Consulta 30/08/2018).

9. Toala B., Zoyla L; Santana Sardi Gustavo; Toala Vera Kerly L. (2021). La inserción de las Tic en el proceso de enseñanza y aprendizaje del contexto universitario ecuatoriano. South Florida Journal of Development, Miami, vol.2 No.2 apr./jun.2021.

10. Zerquera Rodríguez, J. (cols.) (2015). "Multimedia para profesores y estudiantes de 5to año de medicina". Asignatura Salud Pública. MediSur, Universidad de Ciencias Médicas de Cienfuegos Cienfuegos, Cuba 13 (2), (p.254-274). 